\title{
ON THE LIMITATIONS OF LOCALLY ROBUST POSITIVE REDUCTIONS*
}

\author{
LANE A. HEMACHANDRA ${ }^{\dagger}$ \\ Department of Computer Science \\ University of Rochester \\ Rochester, NY 14627, USA \\ SANJAY JAIN $\ddagger$ \\ Department of Computer and Information Sciences \\ University of Delaware \\ Newark, DE 19716, USA
}

\begin{abstract}
Polynomial-time positive reductions, as introduced by Selman, are by definition globally robust - they are positive with respect to all oracles. This paper studies the extent to which the theory of positive reductions remains intact when their global robustness assumption is removed. We note that two-sided locally robust positive reductions - reductions that are positive with respect to the oracle to which the reduction is made - are sufficient to retain all crucial properties of globally robust positive reductions. In contrast, we prove absolute and relativized results showing that one-sided local robustness fails to preserve fundamental properties of positive reductions, such as the downward closure of $N P$.
\end{abstract}

Keywords: Structural complexity theory; Polynomial-time reductions; Complexity classes.

\section{Introduction}

In this paper we study the relative powers of different positive reducibilities. Informally, a reduction is positive if converting some "no" answers to "yes" does not cause a previously accepted string to be rejected.

Selman, in his influential paper [19], defines and considers the properties of polynomial-time positive reductions. His positive reductions are by definition globally

\footnotetext{
* Some of these results were announced at the 9th Conference on Foundations of Software Technology and Theoretical Computer Science, Bangalore, India, 1989.

†Supported in part by the National Science Foundation under grant CCR-8809174/CCR8996198 and Presidential Young Investigator Award CCR-8957604.

${ }^{\ddagger}$ Supported in part by the National Science Foundation under grant CCR-8320136. Work done in part while at the University of Rochester.
} 
robust in the positivity.

An oracle machine, or a set of oracle machines, is said to robustly have a property $P$ if it has property $\mathrm{P}$ for all oracles $[16,10]$; this notion is related to two important early papers by Book, Long and Selman [6,7], which give several careful analyses of properties that hold for all oracles versus properties that hold relative to a fixed oracle. Research on the power of robustness $[16,3,14,20,10]$ suggests that global robustness is a strong restriction. For example, it is known that if two nondeterministic machines $N_{1}$ and $N_{2}$ are robustly complementary - that is, complementary for every oracle - then for all oracles $A, L\left(N_{1}^{A}\right) \in P^{A \oplus N P}[10]$. This, and the desire to broaden the domain of application of Selman's techniques, motivate us to relax the global robustness restriction.

Accordingly, we introduce three notions of locally robust polynomial-time positive reductions. We show that the Turing versions of these reducibilities differ. However, our ability to distinguish among the truth-table versions of these reducibilities depends on the structure of $N P$. In particular, we show that if $P=N P$ then these polynomial-time locally robust truth-table reducibilities are the same. However, if there exist uniformly $\log ^{*}$-sparse tally sets in $N P-P$ then the reducibilities differ.

We study the extent to which the theory of positive reductions, as studied by Selman, remains intact for locally robust reductions. We prove results identifying the crucial properties of positive reductions required to obtain the results of [19]. One reason for introducing new reducibilities is that it is more likely that a set $A$ reduces to $B$ by locally positive reductions than by globally positive reductions. Our results thus enrich the domain in which Selman's techniques can be applied.

\section{Notations}

Let $\mathcal{N}$ denote the set of natural numbers. $\Sigma$ is an alphabet set, usually $\{0,1\}$. A language is a subset of $\Sigma^{*}$. $\emptyset$ denotes the empty set. $M_{0}, M_{1}, \ldots$ denotes some standard enumeration of polynomial-time deterministic Turing machines. $N_{1}, N_{2}, \ldots$ denotes some standard enumeration of polynomial-time nondeterministic Turing machines. We assume that the running time of machine $M_{i}\left(N_{i}\right)$ is bounded by deterministic (nondeterministic) time $n^{i}+i . P$ denotes the class of all languages accepted by some polynomial-time deterministic Turing machine [12]. NP denotes the class of languages accepted by some polynomial-time nondeterministic Turing machine. coNP denotes the class of languages whose complement is in $N P[12] . L(M)$ denotes the language accepted by the machine $M . E$ and $N E$ denote respectively the 
class of languages accepted by exponential time deterministic and nondeterministic Turing machines; that is, $E=\bigcup_{c>0} \operatorname{DTIME}\left[2^{c n}\right]$ and $N E=\bigcup_{c>0} \operatorname{NTIME}\left[2^{c n}\right]$. $L\left(M^{A}\right)$ denotes the language accepted by the oracle machine $M$ with oracle $A$ [12].

$A \leq_{T} B$ means there exists a machine $M$ such that $A=L\left(M^{B}\right) . \leq_{T}^{P}$ denotes polynomial-time Turing reduction. $\leq_{t t}^{P}$ and $\leq_{m}^{P}$ similarly denote polynomialtime truth-table and many-one reductions. $P_{r}(A)$ denotes the class of languages $r$-reducible to $A$ in polynomial time (see [5]). A tally language is a subset of $1^{*} . \bar{A}$ denotes the complement of $A$, i.e., $\Sigma^{*}-A . \chi_{A}$ denotes the characteristic function of $A$. We sometimes denote a string $x$ of length $n$ by $x_{1} x_{2} \cdots x_{n}$, where $x_{i}$ is the $i$-th character of $x .|x|$ denotes the length of $x . A^{\leq n}$ denotes the set of strings in $A$ with length at most $n$.

\section{Polynomial-time Positive Reducibilities}

In this section we review Selman's notion of positive reducibility, which by definition is globally robust, and we introduce the notion of locally robust positive reducibility.

Positive reducibility was first studied for polynomial-time truth-table reductions in [15]. Selman, in [19], extended the definition to Turing reductions. We first give the definition of globally positive reducibility due to Selman. ${ }^{a}$

Definition $3.1[19,18]$ A query machine $M$ is globally positive if $(\forall x)(\forall A, B)[x \in$ $\left.L\left(M^{B}\right) \Rightarrow x \in L\left(M^{A \cup B}\right)\right]$.

Intuitively, a machine $M$ is positive if converting some "no" answers to "yes" answers does not make the machine reject a previously accepted string. Moreover, this property holds for all oracles given to the machine (hence the term globally positive). Positive reducibility can now be defined using these globally positive machines.

Definition $3.2[19,18] A \leq_{\text {pos }}^{P} C$ if $A \leq_{T} C$ by some polynomial-time, globally positive Turing machine $M$.

The conditions placed here on positive Turing reductions are analogous to those in the definition of positive truth-table reductions in [15], which defined globally positive truth-table reductions.

\footnotetext{
${ }^{a}$ This reducibility is simply referred to as "positive" in [19]. However, we shall refer to it throughout this paper as "globally positive" in order to distinguish it from the locally positive reducibilities we define.
} 
Definition 3.3 [15] $A \leq_{p t t}^{P} C$ if $A \leq_{T} C$ by some polynomial-time, globally positive machine $M$, and there is a polynomial-time computable function $f:\{0,1\}^{*} \rightarrow$ $\{c, 0,1,\}^{*}$ such that $M$ on input $x$ makes queries only from the list $f(x)$ (here $c$ acts as a separator of elements of the list). $M$ above can be equivalently represented by a polynomial-time evaluator $e$ such that for all oracles $C, e\left(x, \chi_{C}\left(y_{1}\right), \chi_{C}\left(y_{2}\right), \ldots\right)=$ $M^{C}(x)$, where $y_{1}, y_{2}, \ldots$ are the elements in the list $f(x){ }^{b}$

Definition 3.4 [15] $A \leq_{p b t t}^{P} C$ if $A \leq_{T} C$ by some polynomial-time, globally positive machine $M$, and a polynomial-time computable function $f:\{0,1\}^{*} \rightarrow$ $\{c, 0,1,\}^{*}$ such that $M$ on input $x$ makes queries only from the list $f(x)$. Moreover the number of elements in the list $f(x)$ is bounded by some constant independent of $x$. $M$ above can be equivalently represented by a polynomial-time evaluator $e$ such that for all oracles $C, e\left(x, \chi_{C}\left(y_{1}\right), \chi_{C}\left(y_{2}\right), \ldots\right)=M^{C}(x)$, where $y_{1}, y_{2}, \ldots$ are the elements in the list $f(x)$.

The above definitions require global robustness; given any oracle $A, L\left(M^{A}\right)$ must never decrease when $A$ is increased in any way. Note that all $\leq_{m}^{P}$ reductions are globally positive. However, global robustness is a strong restriction on Turing transducers. Machines exhibiting global robustness are known, in other contexts, to be weak $[3,20,10]$.

A more moderate definition of "positive" might require a reduction to be robust only with respect to the particular set to which the reduction is being made. We introduce three notions of locally robust positive reductions. In these definitions we require the machine to be robust only with respect to the oracle to which the reduction is made.

Definition 3.5 A query machine $M$ is locally right positive with respect to $B$ if $(\forall x)(\forall A)\left[x \in L\left(M^{B}\right) \Rightarrow x \in L\left(M^{A \cup B}\right)\right]$.

Intuitively, $M$ is locally right robust with respect to $B$ if converting some "no" answers from the oracle $B$ to "yes" answers does not make the machine reject a previously accepted string. Left robustness is just the other side of the above definition.

Definition 3.6 A query machine $M$ is locally left positive with respect to $B$ if $(\forall x)(\forall A)\left[x \in L\left(M^{B-A}\right) \Rightarrow x \in L\left(M^{B}\right)\right]$ (or equivalently $(\forall x)(\forall A)\left[x \notin L\left(M^{B}\right) \Rightarrow\right.$ $\left.\left.x \notin L\left(M^{B-A}\right)\right]\right)$.

\footnotetext{
${ }^{b}$ In [15] the first argument of $e$ is $\alpha(x)$, however without loss of generality we can take this to be $x$ and let $e$ do the (polynomial-time) computation required to obtain $\alpha$.
} 
Definition 3.7 A query machine $M$ is locally right-left positive with respect to $B$ if $M$ is both right and left positive with respect to $B$.

Locally robust reductions can now be defined with respect to reductions involving locally robust machines.

Definition 3.8 $A \leq_{\text {rpos }}^{P} B$ if $A \leq_{T} B$ by some polynomial-time machine that is locally right positive with respect to $B$.

Definition 3.9 $A \leq_{l p o s}^{P} B$ if $A \leq_{T} B$ by some polynomial-time machine that is locally left positive with respect to $B$.

Definition 3.10 $A \leq_{\text {rlpos }}^{P} B$ if $A \leq_{T} B$ by some polynomial-time machine that is locally right-left positive with respect to $B$.

$\leq_{r l p t t}^{P}, \leq_{r p t t}^{P}, \leq_{l p t t}^{P}, \leq_{r l p b t t}^{P}, \leq_{r p b t t}^{P}$ and $\leq_{l p b t t}^{P}$ reductions can be defined similarly.

\section{Relationships between Different Polynomial- time Positive Reducibilities}

In this section, we compare the relative power of different polynomial-time positive reducibilities. Clearly:

Proposition $4.1 A \leq_{s}^{P} B \Rightarrow A \leq_{r l s}^{P} B \Rightarrow\left[A \leq_{r s}^{P} B \wedge A \leq_{l s}^{P} B\right]$, where $s$ is in $\{$ pos, ptt, pbtt\}.

We first consider the elementary properties of the reductions. The following proposition is easy to prove.

\section{Proposition 4.2}

1. $A \leq_{\text {pos }}^{P} B$ and $B \leq_{\text {pos }}^{P} C \Rightarrow A \leq_{\text {pos }}^{P} C$.

2. $A \leq_{\text {rpos }}^{P} B$ and $B \leq_{\text {rpos }}^{P} C \Rightarrow A \leq_{\text {rpos }}^{P} C$.

3. $A \leq_{\text {lpos }}^{P} B$ and $B \leq_{\text {lpos }}^{P} C \Rightarrow A \leq_{\text {lpos }}^{P} C$.

4. $A \leq_{\text {rlpos }}^{P} B$ and $B \leq_{\text {rlpos }}^{P} C \Rightarrow A \leq_{\text {rlpos }}^{P} C$.

Results similar to those of Proposition 4.2 can also be proved for bounded truthtable and truth-table reductions.

Proposition $4.3 A \leq_{l p o s}^{P} B \Rightarrow \bar{A} \leq_{r p o s}^{P} \bar{B}$. 
Proof Let $A \leq_{l p o s}^{P} B$ via $M$. Let $M_{1}$ be such that $M_{1}^{C}(x)=1$ iff $M^{\bar{C}}(x)=0$. Clearly $x \in L\left(M_{1}^{\bar{B}}\right) \Leftrightarrow x \notin L\left(M^{B}\right)$. Thus $M_{1}$ reduces $\bar{A}$ to $\bar{B}$. If $C \supseteq \bar{B}$ and $x \in \bar{A}$ (and thus $\bar{C} \subseteq B$ and $x \notin A$ ) then $x \notin L\left(M^{\bar{C}}\right)$, since $M$ is locally left positive, and thus $x \in L\left(M_{1}^{C}\right)$. So $M_{1}$ is locally right positive.

A similar proof can be used for $\leq_{p o s}^{P}, \leq_{r p o s}^{P}, \leq_{r l p o s}^{P}, \leq_{p t t}^{P}, \leq_{l p t t}^{P}, \leq_{r p t t}^{P}, \leq_{r l p t t}^{P}$, yielding the following result.

\section{Proposition 4.4}

1. $A \leq_{\text {rpos }}^{P} B \Rightarrow \bar{A} \leq_{\text {lpos }}^{P} \bar{B}$.

2. $A \leq_{\text {rlpos }}^{P} B \Rightarrow \bar{A} \leq_{\text {rlpos }}^{P} \bar{B}$.

3. (implicit in [19]) $A \leq_{\text {pos }}^{P} B \Rightarrow \bar{A} \leq_{\text {pos }}^{P} \bar{B}$.

4. $A \leq_{l p t t}^{P} B \Rightarrow \bar{A} \leq_{r p t t}^{P} \bar{B}$.

5. $A \leq_{r p t t}^{P} B \Rightarrow \bar{A} \leq_{l p t t}^{P} \bar{B}$.

6. $A \leq_{r l p t t}^{P} B \Rightarrow \bar{A} \leq_{r l p t t}^{P} \bar{B}$.

7. ([15], Proposition $3.1(\mathrm{v})) A \leq_{p t t}^{P} B \Rightarrow \bar{A} \leq_{p t t}^{P} \bar{B}$.

We now consider the relative power of different locally robust positive reductions. Selman showed that globally robust positive Turing reductions are more powerful than truth-table reductions.

Theorem 4.5 [19] There exist recursive sets $A$ and $B$ such that $A \leq_{\text {pos }}^{P} B$ but $A \not t_{t t}^{P} B$.

Also, it is easy to see as a corollary of previous work on disjunctive reductions that (i) there exist recursive sets $A$ and $B$ such that $A \leq_{p t t}^{P} B$ but $A \not_{b t t}^{P} B$, and (ii) there exist recursive sets $A$ and $B$ such that $A \leq_{p b t t}^{P} B$ but $A \not_{m}^{P} B$ [15].

Though locally robust positive reductions are in general more flexible than globally robust positive reductions, the following theorem shows that local robustness does not add extra power for the special case of positive bounded truth-table reductions.

Theorem 4.6 For all $A, P_{p b t t}(A)=P_{r p b t t}(A)=P_{l p b t t}(A)$.

Proof Let $B \leq_{r p b t t}^{P} A$ via $M$. Let $f(x)$ be the polynomial-time computable list such that $M$, on input $x$, makes queries only from the list $f(x)$. Let $e$ be the evaluator equivalent to $M$ (as in the definition of $\leq_{p b t t}^{P}$ reduction). Recall that this means that the size of list $f(x)$ is bounded by some constant $c$ and $e$ is positive with respect to $A$. Thus if $f(x)=x_{1}, x_{2}, \ldots, x_{c}, \chi_{A}\left(x_{i}\right)=b_{i}$ and $e\left(x, b_{1}, \ldots, b_{c}\right)=1$, 
then converting some of $b_{i}$ from 0 to 1 does not make $e$ evaluate to 0 . To make a globally robust reduction from $B$ to $A$ we need to convert this $e$ to $e^{\prime}$ that is positive with respect to all oracles. We do this by converting some evaluation of $e$ from 1 to 0 .

Let $e^{\prime}\left(x, b_{1}, \ldots, b_{c}\right)=1$ iff $\left(\forall d_{1}, \ldots, d_{c}, b_{j}=1 \Rightarrow d_{j}=1\right)\left[e\left(x, d_{1}, \ldots, d_{c}\right)=1\right]$. (For example: if $c=2, e(x, 0,0)=1, e(x, 0,1)=1, e(x, 1,0)=0$ and $e(x, 1,1)=1$ then we replace $e$ by $e^{\prime}$, where $e^{\prime}(x, 0,0)=0, e^{\prime}(0,1)=1, e^{\prime}(x, 1,0)=0$ and $e^{\prime}(x, 1,1)=1$.) This makes $e^{\prime}$ globally positive, and does not effect the reduction from $B$ to $A$ (since $e$ was right positive with respect to $\mathrm{A}$ ). Thus, $f$ and $e^{\prime}$ form a positive bounded truth-table reduction from $B$ to $A$. A similar proof can be used to show that $P_{l p b t t}(A)=P_{p b t t}(A)$.

For unbounded truth-table reductions, the distinction between different positive reducibilities depends on the structure of $N P$, as shown by the following two theorems.

Theorem 4.7 If $P=N P$, then for all $A, P_{p t t}(A)=P_{r l p t t}(A)=P_{r p t t}(A)=P_{l p t t}(A)$.

Theorem 4.8 Let $g(0)=1, g(n+1)=2^{g(n)}, n>0$. If there exist tally sets in $\bigcup_{c>0} N T I M E\left[g^{c}(n)\right]-\bigcup_{c>0} D T I M E\left[g^{c}(n)\right]$ then there is a recursive set $A$ such that $P_{r p t t}(A)-P_{l p t t}(A) \neq \emptyset$ and $P_{l p t t}(A)-P_{r p t t}(A) \neq \emptyset$.

Proof (of Theorem 4.7) We prove that $P_{r p t t}(A)=P_{p t t}(A)$. The proof for $P_{l p t t}(A)=P_{p t t}(A)$ is similar. $P_{r l p t t}(A)=P_{p t t}(A)$ follows from Proposition 4.1.

Let $B \leq_{r p t t}^{P} A$ via $M$. Let $f(x)$ be the polynomial-time computable list such that $M$, on input $x$, makes queries only from the list $f(x)$. Let $e$ be the evaluator equivalent to $M$ (as in the definition of $\leq_{p t t}^{P}$ reduction). We now proceed as in Theorem 4.6. Let $e^{\prime}\left(x, b_{1}, b_{2}, \ldots, b_{p(n)}\right)=1$ iff $\left[\left(\forall d_{1}, \ldots, d_{p(n)}, b_{j}=1 \Rightarrow d_{j}=1\right)\right.$ $\left.\left[e\left(x, d_{1}, \ldots, d_{p(n)}\right)=1\right]\right]$. Note that $e^{\prime}$ can be calculated in polynomial time if $P=N P$. Clearly, $f$ and $e^{\prime}$ witness that $B \leq_{p t t}^{P} A$.

Proof (of Theorem 4.8) We only prove that $(\exists A)\left[P_{r p t t}(A)-P_{l p t t}(A) \neq \emptyset\right]$. The proof can be easily modified to show that $\left[(\exists A)\left[P_{r p t t}(A)-P_{l p t t}(A) \neq \emptyset \wedge P_{l p t t}(A)-\right.\right.$ $\left.\left.P_{\text {rptt }}(A) \neq \emptyset\right]\right]$.

Let $N$ be a polynomial-time nondeterministic machine accepting a tally language $L \subseteq\left\{1^{g(k)}: k \in \mathcal{N}\right\}$ that is not in $P$ (the existence of such a machine 
follows from the assumption that there exist tally sets in $\bigcup_{c>0} \operatorname{NTIME}\left[g^{c}(n)\right]-$ $\bigcup_{c>0} D T I M E\left[g^{c}(n)\right]$, by the techniques of $\left.[11]^{c}\right)$.

Without loss of generality, let all certificates of $x \in L$ be of length $|x|^{j}+j$ and, without loss of generality, $0^{|x|^{j}+j}$ is never such a certificate. Let $r$ be the polynomialtime predicate associated with $N$ and $L$, i.e., $r(x, y)=1$ iff $y$ is a certificate for $x$. Let $e\left(x, y_{1}, \ldots, y_{n^{j}+j}\right)=1-r(x, y)$, where $y=y_{1} \cdots y_{n^{j}+j}$. Let $p l u s(a, j)$ be the string $j$ greater than $a$ in standard lexicographical order; e.g., plus $(1010,3)=1101$. Let $c$ be the separation character from the definition of $f$ (see Definitions 3.3 and 3.4). Let $f(x)=\operatorname{plus}(x, 1) \operatorname{cplus}(x, 2) c \ldots c \operatorname{plus}\left(x,|x|^{j}+j\right)$. Clearly, functions $f$ and $e$ are computable in polynomial time.

$A$ will be defined so that $e$ is locally right positive. Also all strings not of the form $x, \operatorname{plus}(x, 1), \operatorname{plus}(x, 2), \ldots, \operatorname{plus}\left(x,|x|^{j}+j\right)$, where $x \in\left\{1^{g(k)}: k \in \mathcal{N}\right\}$, are not in $A . \chi_{A}(\operatorname{plus}(x, 1)) \cdots \chi_{A}\left(\operatorname{plus}\left(x,|x|^{j}+j\right)\right)$ will be $0^{|x|^{j}+j}$ if $x \notin L$, and otherwise will be a certificate of the fact that $x \in L$. Let $R_{i}$ be the requirement that $M_{i}: \bar{L} \not_{l p t t}^{P} A$, that is, $M_{i}$ does not $\leq_{l p t t}^{P}$ reduce $\bar{L}$ to $A$. Below, $A_{s}$ denotes the strings of $A$ determined before stage $s$. Go to stage 0 .

Stage $s$

1. Let $x$ be the least element in $\left\{1^{g(k)}: k \in \mathcal{N}\right\}$ not considered until this stage.

2. Let $i$ be the least requirement not satisfied until now.

3. Let $e_{i}, f_{i}$ be the evaluator and set calculator (as in the definition of positive truth-table reducibility) for the truth-table reducer $M_{i}$.

4. If $x \notin L$ then let $A_{s+1}=A_{s}$.

5. Else If $(\exists z)\left[z\right.$ is a certificate for $x$ and $\left.e_{i}\left(x, \chi_{D(z)}\left(q_{1}\right), \chi_{D(z)}\left(q_{2}\right), \ldots\right)=1\right]$, where $f_{i}(x)=q_{1} c q_{2} \cdots$ and $D(z)=A_{s} \bigcup\left\{p l u s(x, l): z_{l}=1\right\}$, then let $y$ be the least such certificate $z$. Set $A_{s+1}=A_{s} \bigcup\left\{p l u s(x, l): y_{l}=1\right\}$. (Note that here $R_{i}$ is satisfied.)

6. Else Let $A_{s+1}=A_{s} \bigcup\left\{p l u s(x, l): y_{l}=1\right\}$, where $y$ is the least certificate for $x$. (Note that besides the explicit satisfaction of $R_{i}$ in step $5, R_{i}$ may be implicitly satisfied due to steps 4 and 6.)

end stage $s$

\footnotetext{
${ }^{c}$ Though recent work by Allender [1] has corrected parts of [11], the techniques of [11] as used here are correct.
} 
It is clear that $\bar{L} \leq_{r p t t}^{P} A$ via the functions $f$ and $e$. This is because when $x \in \bar{L}$, then $e(x, y)=1$ for any length $|x|^{i}+i$ string $y$; so even if $A$ has some strings added - and the "address" $\chi_{A}(\operatorname{plus}(x, 1)) \cdots \chi_{A}\left(\operatorname{plus}\left(x,|x|^{i}+i\right)\right)=0^{|x|^{i}+i}$ thus has some zeros corrupted to ones $-e(x$, corrupted address) will nonetheless accept.

Now consider the following cases:

Case 1: All requirements are satisfied.

In this case, clearly, $\bar{L} \not_{l p t t}^{P} A$.

Case 2: $R_{i}$ is the least requirement not satisfied.

In this case we show that $L \in P$. Let $n$ be so large that $2^{n / 10}>n^{i}+i$, and all the smaller requirements have been satisfied before stage $s, n>g(s)$. Clearly, when $m \in\{g(k): k \in \mathcal{N}\}$, then $A^{\leq m-1}$ can be determined in time polynomial in $m$ (by just going through all possible certificates). Now for $x \in\left\{1^{g(k)}: k \in \mathcal{N}\right\},|x|>n$, we have $x \notin L \Rightarrow e_{i}\left(x, \chi_{A \leq|x|-1}\left(q_{1}\right), \chi_{A \leq|x|-1}\left(q_{2}\right), \ldots\right)=1$ (since $A \leq|x|-1=A \leq|x|^{j}+j$ due to step 4 of the construction, and $M_{i}$ reduces $\bar{L}$ to $A$ ). And similarly, we have $x \in L \Rightarrow e_{i}\left(x, \chi_{A \leq|x|-1}\left(q_{1}\right), \chi_{A \leq|x|-1}\left(q_{2}\right), \ldots\right)=0$ (since $A \leq|x|-1 \subseteq A$ and $\bar{L} \leq_{l p t t}^{P} A$ via $\left.M_{i}\right)$. This gives us a polynomial-time decision procedure for $L$, contradicting the assumption.

Thus all requirements are satisfied.

Note that the above proof can also be used to distinguish between $\leq_{r p t t}^{P}$ and $\leq_{\text {lpos }}^{P}$ reductions, under the same assumption.

We now consider the relationship between various positive Turing reducibilities.

Theorem $4.9(\exists A)\left[P_{\text {rpos }}(A)-P_{\text {lpos }}(A) \neq \emptyset \wedge P_{\text {lpos }}(A)-P_{\text {rpos }}(A) \neq \emptyset\right]$.

Corollary $4.10(\exists A)\left[P_{\text {rpos }}(A)-P_{\text {rlpos }}(A) \neq \emptyset \wedge P_{\text {lpos }}(A)-P_{\text {rlpos }}(A) \neq \emptyset\right]$.

Proof Let $g(0)=1, g(n+1)=2^{g(n)}$. Consider the following languages:

$L_{A}=\left\{1^{n}: n=g(k)\right.$ for some even $k \wedge 1^{n} b_{0} b_{1} b_{2} \cdots b_{n-1} \in A$ where $b_{j}=$ $\left.\chi_{A}\left(0^{n+j}\right)\right\}$.

$L_{A}^{\prime}=\left\{1^{n}: n=g(k)\right.$ for some odd $k \wedge 1^{n} b_{0} b_{1} b_{2} \cdots b_{n-1} \in A$ where $b_{j}=$ $\left.\chi_{A}\left(0^{n+j}\right)\right\}$.

To ensure that $L_{A} \leq_{r \text { pos }}^{P} A$, it suffices to construct $A$ so that for all $n$ of the form $g(2 k)$, we have $\left[\left[1^{n} b_{0} b_{1} \cdots b_{n-1} \in A\right] \bigwedge\left[(\forall j)\left[b_{j}=1 \Rightarrow d_{j}=1\right]\right]\right]$ $\Rightarrow\left[1^{n} d_{0} d_{1} \cdots d_{n-1} \in A\right]$, where $b_{j}=\chi_{A}\left(0^{n+j}\right)$. Thus an oracle machine $M^{B}$ that accepts $1^{n}$ iff $n=g(k)$ for some even $k$ and $1^{n} a_{0} a_{1} \cdots a_{n-1} \in B$, where $a_{i}=\chi_{B}\left(0^{n+i}\right)$ witnesses that $L_{A} \leq_{r p o s}^{P} A$. 
Similarly, $L_{A}^{\prime} \leq_{l p o s}^{P} A$ is ensured if for all $n$ of the form $g(2 k+1)$, for $b_{j}=$ $\chi_{A}\left(0^{n+j}\right),\left[\left[1^{n} b_{0} b_{1} \cdots b_{n-1} \notin A\right] \bigwedge(\forall j)\left[b_{j}=0 \Rightarrow d_{j}=0\right]\right] \Rightarrow\left[1^{n} d_{0} d_{1} \cdots d_{n-1} \notin A\right]$.

We now construct $A$ in stages. $A$ will satisfy the conditions above so that $L_{A} \leq_{r p o s}^{P} A$ and $L_{A}^{\prime} \leq_{l p o s}^{P} A$.

At stage $s$ we decide the membership in $A$ of strings of length $g(s), \ldots, g(s+1)-1$. We always assume that strings not of the form $0^{g(k)+i}, i<g(k)$ or $1^{g(k)}\{0,1\}^{g(k)}$, are not in $A$ (without explicitly mentioning it below).

Let $R_{2 i}$ be the requirement that $M_{i}: L_{A} \mathbb{Z}_{\text {lpos }}^{P} A$, that is, $M_{i}$ does not $\leq_{\text {lpos }}^{P}$ reduce $L_{A}$ to $A$. Let $R_{2 i+1}$ be the requirement that $M_{i}: L_{A}^{\prime} \mathbb{Z}_{r p o s}^{P} A$, that is, $M_{i}$ does not $\leq_{r p o s}^{P}$ reduce $L_{A}^{\prime}$ to $A$. Note that if all the requirements are satisfied then $L_{A} \mathbb{Z}_{\text {lpos }}^{P} A$ and $L_{A}^{\prime} \mathbb{Z}_{\text {rpos }}^{P} A$. Below, $A_{s}$ denotes the strings of $A$ determined before stage $s$. Go to stage 0 .

$\underline{\text { Stage } 2 s}$

1. Let $R_{2 i}$ be the least unsatisfied even requirement.

2. Let $n=g(2 s)$.

3. If $2^{n / 10} \leq n^{i}+i$ then exclude from $A$ all strings of length $l, g(2 s) \leq l<$ $g(2 s+1)$.

4. Else If $M_{i}^{A}\left(1^{n}\right)$ rejects when all new questions $x$ (i.e., those not decided in $\left.A_{2 s}\right)$ are answered by the rule "If $x$ is of the form $1^{n}\{0,1\}^{n}$ then YES; If $x$ is of the form $0^{n+j}$ then $N O$," then let $A_{2 s+1}$ be such that all strings of the form $1^{n}\{0,1\}^{n} \in A_{2 s+1}$ and all other strings of length $l, g(2 s) \leq l<g(2 s+1)$ not in $A_{2 s+1}$. (Note that in this case $R_{2 i}$ is satisfied.)

5. Else

- Let $S$ be the set of questions of the form $1^{n}\{0,1\}^{n}$ asked in the computation by $M_{i}$ in step 4 above.

- For all $x \in S$, let $x \in A$.

- If $x$ is of the form $1^{n}\{0,1\}^{n}$ and $x \notin S$ then let $x \notin A$.

- Let $y$ be a question of the form $1^{n}\{0,1\}^{n}$ not asked by $M_{i}$ (there exists such a $y$ ).

- Let $0^{n+j} \in A \Leftrightarrow y_{n+j+1}=1$ for $j<n$. 
- (Note that on this $A, M_{i}$ either accepts incorrectly or rejects. In the latter case, since $A \supseteq A_{2 s} \bigcup S$ on which $M_{i}$ accepts, $M_{i}$ is not a $\leq_{l p o s}^{P}$ reduction. Either way, $R_{2 i}$ is satisfied.)

end stage $2 s$

Stage $2 s+1$ is similar:

Stage $2 s+1$

1. Let $R_{2 i+1}$ be the least unsatisfied odd requirement.

2. Let $n=g(2 s+1)$.

3. If $2^{n / 10} \leq n^{i}+i$ then exclude from $A$ all strings of length $l, g(2 s+1) \leq l<$ $g(2 s+2)$.

4. Else If $M_{i}^{A}\left(1^{n}\right)$ accepts when all new questions $x$ (i.e., those not decided in $\left.A_{2 s}\right)$ are answered by the rule "If $x$ is of the form $1^{n}\{0,1\}^{n}$ then NO; If $x$ is of the form $0^{n+j}$ then YES," then let $A_{2 s+1}$ be such that all strings of the form $1^{n}\{0,1\}^{n} \notin A_{2 s+2}$ and all strings of the form $0^{n+j}, j<n$, in $A_{2 s+2}$. (Note that in this case $R_{2 i+1}$ is satisfied.)

5. Else

- Let $S$ be the set of questions of the form $1^{n}\{0,1\}^{n}$ asked in the above computation by $M_{i}$.

- For all $x \in S$, let $x \notin A$.

- If $x$ is of the form $1^{n}\{0,1\}^{n}$ and $x \notin S$ then let $x \in A$.

- Let $y$ be a question of the form $1^{n}\{0,1\}^{n}$ not asked by $M_{i}$ (there exists such a $y$ ).

- Let $0^{n+j} \in A \Leftrightarrow y_{n+j+1}=1$ for $j<n$.

- Note that on this $A$, either $M_{i}$ rejects incorrectly, or $M_{i}$ accepts. In the latter case, since $A \subseteq A_{2 s} \bigcup\left\{0^{n+j}: j<n\right\} \bigcup\left\{1^{n} z:|z|=n, z \notin S\right\}$ on which $M_{i}$ rejects, $M_{i}$ is not a $\leq_{r p o s}^{P}$ reduction. In either case, $R_{2 i+1}$ is satisfied.)

end stage $2 s+1$

Let $M^{B}\left(1^{n}\right)=1$ iff $n=g(2 k)$ for some $k$ and $1^{n} b_{0} b_{1} \cdots b_{n-1} \in B$, where $b_{j}=1$ iff $0^{n+j} \in B$. Clearly, $L_{A} \leq_{r p o s}^{P} A$ via $M$ (since $1^{n}$ is placed in $L_{A}$ only in step 4 
in which case all strings of the form $1^{n} z,|z|=n$, are also placed in $A$ ). Similarly $L_{A}^{\prime} \leq_{\text {lpos }}^{P} A$. We claim that $L_{A}{\underset{Z}{l p o s}}_{\text {lpos }} A$. Suppose by way of contradiction that $L_{A} \leq_{\text {lpos }}^{P} A$ via $M_{i}$. Also let $M_{i}$ be the least such machine. Then for sufficiently large $s$ in stage $2 s, 2^{n / 10}>n^{i}+i$ and all smaller even requirements have been satisfied. Thus at this stage by construction $M_{i}$ will be fooled. Thus no such machine can exist. It can be similarly shown that $L_{A}^{\prime} \mathbb{Z}_{\text {rpos }}^{P} A$. This proves the theorem.

Theorem $4.11(\exists A)\left[P_{\text {rpos }}(A) \cap P_{\text {lpos }}(A) \neq P_{\text {rlpos }}(A)\right]$.

Proof Let $g(0)=1, g(n+1)=2^{g(n)}$. Consider the following languages:

$L_{A}=\left\{1^{n}: n=g(k)\right.$ for some $k \wedge 1^{n} b_{0} b_{1} b_{2} \cdots b_{n-1} \in A$ where $\left.b_{j}=\chi_{A}\left(0^{n+j}\right)\right\}$.

$L_{A}^{\prime}=\left\{1^{n}: n=g(k)\right.$ for some $k \wedge 1^{2 n} b_{0} b_{1} b_{2} \cdots b_{n-1} \in A$ where $b_{j}=$ $\left.\chi_{A}\left(0^{2 n+j}\right)\right\}$.

To ensure that $L_{A} \leq_{\text {rpos }}^{P} A$ it suffices to construct $A$ so that for all $n$ of the form $g(k)$, for $b_{j}=\chi_{A}\left(0^{n+j}\right)$, we have $\left[\left[1^{n} b_{0} b_{1} \cdots b_{n-1} \in A\right] \wedge(\forall j)\left[b_{j}=1 \Rightarrow d_{j}=1\right]\right]$ $\Rightarrow\left[1^{n} d_{0} d_{1} \cdots d_{n-1} \in A\right]$. Thus $L_{A} \leq_{r p o s}^{P} A$ is witnessed by an oracle machine $M^{B}$ that accepts $1^{n}$ iff (1) $n=g(k)$ for some $k$ and $(2) 1^{n} a_{0} a_{1} \cdots a_{n-1} \in B$, where $a_{i}=\chi_{B}\left(0^{n+i}\right)$. Similarly, to ensure that $L_{A}^{\prime} \leq_{l p o s}^{P} A$, it suffices to construct $A$ so that for all $n$ of the form $g(k)$, we have $\left[\left[1^{2 n} b_{0} b_{1} \cdots b_{n-1} \notin A\right] \wedge(\forall j)\left[b_{j}=0 \Rightarrow\right.\right.$ $\left.\left.d_{j}=0\right]\right] \Rightarrow\left[1^{2 n} d_{0} d_{1} \cdots d_{n-1} \notin A\right]$, where $b_{j}=\chi_{A}\left(0^{2 n+j}\right)$. Thus $L_{A}^{\prime} \leq_{l p o s}^{P} A$ is witnessed by an oracle machine $M^{B}$ that accepts $1^{n}$ iff (1) $n=g(k)$ for some $k$ and (2) $1^{2 n} a_{0} a_{1} \cdots a_{n-1} \in B$, where $a_{i}=\chi_{B}\left(0^{2 n+i}\right)$. We will also ensure that $L_{A}=L_{A}^{\prime}$. Thus $L_{A} \in P_{\text {rpos }}(A) \cap P_{\text {lpos }}(A)$. We will also ensure that no machine $M_{i}$ witnesses that $L_{A} \leq_{\text {rlpos }}^{P} A$.

We now construct $A$ in stages. $A$ will satisfy the conditions above so that $L_{A} \leq_{\text {rpos }}^{P} A, L_{A} \leq_{\text {lpos }}^{P} A$ and $L_{A} \mathbb{Z}_{\text {rlpos }} A$.

At stage $s$ we decide the membership in $A$ of strings of lengths $g(s), \ldots, g(s+1)-$ 1. We always assume that strings not of the forms $(1) 0^{g(k)+i}, i<2 g(k)$, or (2) $1^{g(k)}\{0,1\}^{g(k)}$, or $(3) 1^{2 g(k)}\{0,1\}^{g(k)}$ are not in $A$ (without explicitly mentioning it below).

Let $R_{i}$ be the requirement that $M_{i}: L_{A}{\underset{Z}{r l p o s}}_{P}^{P} A$ - that is, $M_{i}$ does not $\leq_{r l p o s}^{P}$ reduce $L_{A}$ to $A$. Note that if all the requirements are satisfied then $L_{A} \not_{\text {rlpos }}^{P} A$. Below, $A_{s}$ denotes the strings of $A$ determined before stage $s$. Go to stage 0 .

Stage $s$

1. Let $R_{i}$ be the least unsatisfied requirement. 
2. Let $n=g(s)$.

3. If $2^{n / 100} \leq n^{i}+i$ then exclude from $A$ all strings of length $l, g(s) \leq l<g(s+1)$.

4. Else If $M_{i}^{A_{s} \cup\left\{1^{n}\{0,1\}^{n}\right\} \cup\left\{0^{n+j}: n / 2 \leq j<n\right\} \cup\left\{0^{2 n+j}: n / 2 \leq j<n\right\}}\left(1^{n}\right)$ rejects then let $y \in 1^{2 n} 0^{n / 2}\{0,1\}^{n / 2}$ be a string such that $y$ is not queried in the above computation. Let $A_{s+1}=A_{s} \cup\left\{1^{n}\{0,1\}^{n}\right\} \cup\left\{0^{n+j}: n / 2 \leq j<n\right\} \cup\{y\} \cup\left\{0^{2 n+j}\right.$ : $\left.y_{2 n+j+1}=1\right\}$.

(Note that in this case $M_{i}$ is fooled since $M_{i}^{A_{s} \cup\left\{1^{n}\{0,1\}^{n}\right\} \cup\left\{0^{n+j}: n / 2 \leq j<n\right\} \cup\left\{0^{2 n+j}: n / 2 \leq j<n\right\} \cup\{y\}}\left(1^{n}\right)$ rejects and $1^{n} \in L_{A}$ and $A_{s+1} \subseteq$ $\left.A_{s} \cup\left\{1^{n}\{0,1\}^{n}\right\} \cup\left\{0^{n+j}: n / 2 \leq j<n\right\} \cup\left\{0^{2 n+j}: n / 2 \leq j<n\right\} \cup\{y\}.\right)$

5. Else $\left(M_{i}^{A_{s} \cup\left\{1^{n}\{0,1\}^{n}\right\} \cup\left\{0^{n+j}: n / 2 \leq j<n\right\} \cup\left\{0^{2 n+j}: n / 2 \leq j<n\right\}}\left(1^{n}\right)\right.$ accepts). Let $y \in$ $1^{n}\{0,1\}^{n / 2} 1^{n / 2}$ be a string such that $y$ is not queried in the above computation. Let $A_{s+1}=\left[A_{s} \cup\left\{1^{n}\{0,1\}^{n}\right\} \cup\left\{0^{2 n+j}: n / 2 \leq j<n\right\} \cup\left\{0^{n+j}: y_{n+j+1}=\right.\right.$ $1\}]-\{y\}$.

(Note that in this case $M_{i}$ is fooled since $M_{i}^{\left[A_{s} \cup\left\{1^{n}\{0,1\}^{n}\right\} \cup\left\{0^{n+j}: n / 2 \leq j<n\right\} \cup\left\{0^{2 n+j}: n / 2 \leq j<n\right\}\right]-\{y\}}\left(1^{n}\right)$ accepts, $1^{n} \notin L_{A}$ and $A_{s+1} \supseteq$ $\left.\left[A_{s} \cup\left\{1^{n}\{0,1\}^{n}\right\} \cup\left\{0^{n+j}: n / 2 \leq j<n\right\} \cup\left\{0^{2 n+j}: n / 2 \leq j<n\right\}\right]-\{y\}.\right)$

end stage $s$

Clearly, $L_{A} \leq_{r p o s}^{P} A$ (since $1^{n}$ is placed in $L_{A}$ only in step 4 in which case all strings of the form $1^{n} z,|z|=n$, are also placed in $A$ ). Similarly $L_{A} \leq_{\text {lpos }}^{P} A$. We claim that $L_{A} \mathbb{Z}_{\text {rlpos }}^{P} A$. Suppose by way of contradiction that $L_{A} \leq_{\text {rlpos }}^{P} A$ via machine $M_{i}$, and, without loss of generality, let $M_{i}$ be the least machine witnessing the reduction. Then for sufficiently large $s$ in stage $s, 2^{n / 100}>n^{i}+i$ and all smaller even requirements have been satisfied. Thus at this stage by construction $M_{i}$ will be fooled. Thus no such machine can exist.

Whether $\leq_{\text {pos }}^{P}$ and $\leq_{r l p o s}^{P}$ are different is at present an open problem.

\section{Basic Properties of Reductions}

In this section we consider some of the basic properties of positive reductions in NP. Selman, in [19], showed that $N P$ is closed downward under globally robust positive Turing reductions. We show that, though Selman's techniques suffice to prove that $N P$ is closed downwards under two of the locally robust reductions, the remaining 
locally robust reduction fails to leave $N P$ closed downwards in some relativized worlds. As a corollary, we note that rpos and lpos reductions do not share the complementation property of globally robust positive reductions (Proposition 4.4, part 3).

Theorem 5.1 NP is closed downward under $\leq_{l p o s}^{P}$ reductions.

\section{Corollary 5.2}

1. coNP is closed downward under $\leq_{r p o s}^{P}$ reductions.

2. $N P$ and $c o N P$ are closed downward under $\leq_{r l p o s}^{P}$ reductions.

3. [19] $N P$ and $c o N P$ are closed downward under $\leq_{\text {pos }}^{P}$ reductions.

Proof Let $A \leq_{l p o s}^{P} B, B \in N P$. We give an $N P$ algorithm for $A$. Let $A \leq_{\text {lpos }}^{P} B$ via $M$.

On input $x$

Simulate $M$, guessing answers for each question asked.

Verify the answers guessed YES.

Accept iff $M$ accepts.

If $x \in A$, then there exists a sequence of right guesses by which the above algorithm accepts.

We now consider the case in which $x \notin A$. Clearly the guessed oracle for which the above algorithm simulates $M$ is a subset of $B$. Since $x \notin M^{B}, x \notin M^{C}$ for all $C \subseteq B$ (since $M$ is locally left positive with respect to $B$ ). Thus the above algorithm does not accept $x$.

However the proof does not work for right positive reductions. We give a relativized world in which $N P$ is not closed downward under locally right robust positive reductions.

Theorem 5.3 There is a recursive oracle $B$ such that $N P^{B}$ is not closed downward under $\leq_{r p o s}^{P}$ reductions. That is, there are recursive sets $A, B$ and $C$ such that $C \leq_{\text {rpos }}^{P} A, A \in N P^{B}$ and $C \notin N P^{B}$.

Proof This proof is similar to the proof of Theorem 4.9. Let $g(0)=$ $1, g(n+1)=2^{g(n)}$. We will define sets $A$ and $B$. Let $A=\{x:(\exists y)|y|=|x|, x y \in B\}$. Clearly $A \in N P^{B}$. Let $L_{A}=\left\{1^{n}: n=g(k)\right.$ for some $k$ and $1^{n} b_{0} b_{1} \cdots b_{n-1} \in A$ where $\left.b_{j}=1 \Leftrightarrow 0^{n+j} \in A\right\}$. If $\left[1^{n} b_{0} b_{1} \cdots b_{n-1} \in A \bigwedge(\forall j)\left[b_{j}=1 \Rightarrow d_{j}=1\right]\right] \Rightarrow$ $\left[1^{n} d_{0} d_{1} \cdots d_{n-1} \in A\right]$, where $b_{j}=1 \Leftrightarrow 0^{n+j} \in A$, then $L_{A} \leq_{r p o s}^{P} A$ (via machine $M$ 
with which oracle $D$ accepts $1^{n}$ iff $n=g(k)$ for some $k$ and $1^{n} a_{0} a_{1} \cdots a_{n-1} \in D$, where $\left.a_{i}=\chi_{D}\left(0^{n+i}\right)\right)$. We will construct $B$ so that $A$ satisfies the above property. In addition we will ensure that $L_{A} \notin N P^{B}$. Taking $C=L_{A}$ proves the theorem.

Let $R_{i}$ be the requirement that $L\left(N_{i}^{B}\right) \neq L_{A}$. A will contain strings of the form $1^{n} z,|z|=n$, and $0^{n+i}, i<n$, where $n=g(k)$ for some $k$ (this thus restricts some elements to be out of $B$; we assume that such elements are not in $B$ without explicitly mentioning so). At stage $s$ we decide the membership of strings of length $l, g(s) \leq l<g(s+1)$ in $A$ (and strings of length $l, 2 g(s) \leq l<2 g(s+1)$ in $B$ ). Below $B_{s}$ denotes the strings of $B$ determined before stage $s$. Go to stage 0 .

$\underline{\text { Stage } s}$

1. Let $R_{i}$ be the least unsatisfied requirement.

2. Let $n=g(s)$.

3. If $2^{n / 10} \leq n^{i}+i$ then exclude from $A$ all strings of length $l, n \leq l<2^{n}$.

4. Else If $N_{i}^{B_{s} \bigcup\left\{0^{2(n+j)}: n>j \geq n / 2\right\} \bigcup\left\{1^{n} z 0^{2 n}:|z|=n, z \geq 0^{n / 2} 1^{n / 2}\right\}}\left(1^{n}\right)$ rejects then let $B_{s+1}=B_{s} \bigcup\left\{0^{2(n+j)}: n>j \geq n / 2\right\} \bigcup\left\{1^{n} z 0^{2 n}:|z|=n, z \geq 0^{n / 2} 1^{n / 2}\right\}$.

(Note that in this case we have already fooled $N_{i}$, since $1^{n} \in L_{A}-L\left(N_{i}^{B}\right)$.)

5. Else

- Fix an accepting path of $N_{i}^{B_{s} \bigcup\left\{0^{2(n+j)}: n>j \geq n / 2\right\} \bigcup\left\{1^{n} z 0^{2 n}:|z|=n, z \geq 0^{n / 2} 1^{n / 2}\right\}}$ running on input $1^{n}$.

- Let $S$ be the set of questions asked by $N_{i}$ that are in $\left\{0^{(n+j)} w:|w|=\right.$ $n+j\} \bigcup\left\{1^{n} z 0^{2 n}:|z|=n, z \geq 0^{n / 2} 1^{n / 2}\right\}$.

- Let $y,|y|=n, y \in\{0,1\}^{n / 2} 1^{n / 2}$ be such that $1^{n} y 0^{2 n} \notin S$ (clearly, such a $y$ exists).

- Let $s_{n+j}$ be a string of length $n+j$ such that $0^{(n+j)} s_{n+j} \notin S$.

- Let $B_{s+1}=B_{s} \bigcup\left\{w: w \in\left\{0^{2(n+j)}: n>j \geq n / 2\right\} \bigcup\left\{1^{n} z 0^{2 n}:|z|=\right.\right.$ $n, z \geq 0^{n / 2} 1^{n / 2}$ and $\left.\left.1^{n} z 0^{2 n} \in S\right\}\right\} \bigcup\left\{0^{(n+j)} s_{n+j}: y_{j+1}=1\right\}$.

- (Note that in this case $1^{n} \in L\left(N_{i}^{B}\right)-L_{A}$.)

$\underline{\text { end stage } s}$

Clearly, $L_{A} \leq_{r p o s}^{P} A$. If all requirements are satisfied then clearly $L_{A} \notin N P^{B}$. So assume that $R_{i}$ is the least requirement not satisfied. Let $s$ be so large that $2^{n / 10}>n^{i}+i$, and all requirements less that $i$ are satisfied by stage $s$. Then by 
construction $R_{i}$ will be satisfied at stage $s$. Thus all the requirements are satisfied.

Though $A \leq_{\text {pos }}^{P} B \Rightarrow \bar{A} \leq_{\text {pos }}^{P} \bar{B}$ (Proposition 4.4), the analog of this result fails for rpos and lpos reductions, as an immediate corollary of Theorems 5.1 and 5.3 and Corollary 5.2.

\section{Corollary 5.4}

1. There exist recursive oracles $A$ and $B$ such that $A \leq_{r p o s}^{P} B$ but $\bar{A} \bigsqcup_{\text {rpos }}^{P} \bar{B}$.

2. There exist recursive oracles $A$ and $B$ such that $A \leq_{\text {lpos }}^{P} B$ but $\bar{A} \not_{\text {lpos }}^{P} \bar{B}$.

\section{$6 \quad$ P-Selectivity and Positive Reductions}

Selman, in [17], introduced the notion of $P$-selectivity. Intuitively, $A$ is $P$-selective if, given two strings $x$ and $y$, a polynomial-time function can determine which of $x$ or $y$ is more "likely" to be an element of $A$.

Definition 6.1 [17] $A$ is $P$-selective if there exists a polynomial-time computable function $f$ such that:

1. $(\forall x, y) f(x, y) \in\{x, y\}$, and

2. $x \in A \vee y \in A \Rightarrow f(x, y) \in A$.

Selman [19] showed that if $A \leq_{p o s}^{P} \bar{A}$ and $A$ is $P$-selective then $A$ is in $P$. Selman's proof can be easily seen to generalize to the following:

Theorem 6.2 $A \in P$ if and only if $A \leq_{r l p o s}^{P} \bar{A}$, and $A$ is $P$-selective.

We leave it as an open problem whether $\leq_{\text {rpos }}^{P}$ or $\leq_{\text {lpos }}^{P}$ reducibility suffices to obtain the above theorem. Below, we show that weak $P$-selectivity does not suffice.

Ko [13] generalized Selman's notion of $P$-selectivity.

Definition 6.3 [13] A preorder $R$ on $\Sigma^{*}$ is partially polynomial-time computable if there is a polynomial-time computable function $f$ such that:

1. $f(x, y)=f(y, x)=x$ if $x R y$ but not $y R x$,

2. $f(x, y)=f(y, x) \in\{x, y\}$ if $x R y$ and $y R x$, and

3. $f(x, y)=\#$ otherwise.

Let $x S y$ if and only if $x R y$ and $y R x$. Let $R^{\prime}$ be an induced ordering on $\Sigma^{*} / S$, i.e., $\bar{x} R^{\prime} \bar{y}$ iff $x R y$, where $\bar{x}$ denotes the equivalence class of $x$ under the relation $S$. 
Definition 6.4 [13] A partial ordering $R$ is p-linear if, for all $n$, the set $\Sigma_{n}=$ $\{x:|x| \leq n\}$ can be decomposed into at most $p(n)$ many pairwise disjoint sets $B_{1}, \ldots, B_{m}, m \leq p(n)$, for some polynomial $p$ such that:

1. If $x$ and $y$ are in the same set $B_{i}$ then $x R y \vee y R x$, and

2. if $x$ and $y$ are in two different sets then neither $x R y$ nor $y R x$.

Definition 6.5 [13] $A$ is weakly $P$-selective if and only if there is a partially polynomial-time computable preorder $R$ with the induced equivalence relation $S$ and partial ordering $R^{\prime}$ such that:

1. $R^{\prime}$ is p-linear, and

2. for all $n, A_{n}=\{x \in A:|x| \leq n\}$ is the union of initial segments of $R^{\prime}$ chains in $\Sigma_{n}$.

In contrast to Theorem 6.2 we show that:

Theorem 6.6 There exists recursive oracle $Q$ and a recursive set $A$ such that $A$ is weakly $P^{Q}$-selective, $A \leq_{\text {pos }}^{P} \bar{A}$ but $A \notin P^{Q}$.

Proof We will define $A$ and $Q$ in the following. $Q$ will act as a weak $P$-selector for $A$. Thus $A$ will be trivially weakly $P^{Q}$-selective.

Let $g(0)=1, g(n+1)=8^{g(n)}$. $A$ and $Q$ will be such that:

1. $A \subseteq S$ where $S=\left[\left\{1^{g(n)}: n \in \mathcal{N}\right\} \cup\left\{1^{g(n)} 0^{k}: n \in \mathcal{N} \wedge 0<k \leq 2 g(n)\right\} \cup\right.$ $\left.\left\{1^{4 g(n)} y: n \in \mathcal{N} \wedge|y|=1+g(n)\right\}\right]$.

2. $1^{g(n)} 0^{2 k+1} \in A \Leftrightarrow 1^{g(n)} 0^{2 k+2} \notin A$, for $k<g(n)$.

3. for $|y|=g(n), 1^{4 g(n)} y 1 \in A \Leftrightarrow 1^{4 g(n)} y 0 \notin A$.

4. $1^{g(n)} \in A \Leftrightarrow 1^{4 g(n)} y 1 \in A$ where $y=\chi_{A}\left(1^{g(n)} 0^{2}\right) \chi_{A}\left(1^{g(n)} 0^{4}\right) \cdots \chi_{A}\left(1^{g(n)} 0^{2 g(n)}\right)$.

For partial ordering $R$ we have

1. $B_{n}=\{x:|x|=n\}$ (for $B_{i}$ in the definition of p-linear partial ordering).

2. $\langle x, y\rangle \in Q$ if and only if $|x|=|y|$ and $x R y$.

Clearly, $A \leq_{\text {pos }}^{P} \bar{A}$ and $A$ is weakly $P^{Q}$-selective.

The following construction diagonalizes to ensure that every $P^{Q}$ machine fails to accept $A$. Assume, without loss of generality, that $M_{i}^{Q}$ queries only strings of 
the form $\langle x, y\rangle$ such that $|x|=|y|, x \in S$ and $y \in S$. At stage $s$ we determine the membership in $A$ for all strings of length $l, g(s) \leq l<g(s+1)$. We also define $Q$ on all pairs of strings of length between $g(s)$ and $g(s+1)$. We explicitly give the membership in $A$ only for strings in $S$. Also we define the relation $R$ only for strings in $S$ that are of the same length. $A$ and $R$ on other values can be predetermined using (1) and (5) above.

Let $R_{i}$ be the requirement that $L\left(M_{i}^{Q}\right) \neq A$. Go to stage 0 .

Stage $s$

1. Let $x=1^{g(s)}$.

2. Let $i$ be the least requirement not satisfied until now. Let $n=g(s)$.

3. If $n^{i}+i \geq 8^{n / 10}$ then let $1^{n} \notin A, 1^{n} 0^{2 k} \in A, k \leq n$, and $1^{4 n} y 1 \notin A$ for all $y,|y|=n$. Define $Q$ in some way consistent with $A$.

4. Else run $M^{i}$ on $1^{n}$, answering all questions $\langle z, y\rangle$ in the following way: "If $5 g(s)+1=|z|=|y|$, then let $z=u c, y=w r ; r, c \in\{0,1\} ;$ Put $u 0, w 0 \in A$ and $u 1, w 1 \notin A$; Answer the question in a way consistent with the previous answers and $A$ as determined so far."

5. Let $y$ be such that neither $1^{4 n} y 0$ nor $1^{4 n} y 1$ has appeared in any query until now. Let $\chi_{A}\left(1^{n} 0^{2}\right) \chi_{A}\left(1^{n} 0^{4}\right) \cdots \chi_{A}\left(1^{n} 0^{2 n}\right)=y$.

6. Let $1^{4 n} y 1 \in A$ if and only if $M$ is rejected in the above simulation.

7. (Note that $M_{i}$ has been fooled in this stage.)

end stage $s$

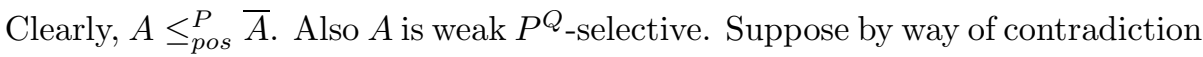
that $M_{i}^{Q}=A$. Also let $M_{i}$ be the least such machine. Then for sufficiently large $s$ in stage $s, 8^{n / 10}>n^{i}+i$ and all smaller requirements are satisfied. Thus at this stage by construction $M_{i}$ will be fooled. Thus no such machine can exist.

Selman [19] showed that if $A \leq_{\text {pos }}^{P} B\left(B \neq \emptyset, B \neq \Sigma^{*}\right)$ and $B$ is $P$-selective, then there exists an algorithm that runs in time polynomial in the number of queries in the computation tree of the reducer and outputs a set $I$ such that $x \in A \Leftrightarrow I \subseteq B$. We observe that Selman's proof holds even for $\leq_{r l p o s}^{P}$ reductions.

Proposition 6.7 [implicit from the techniques of [19]] Let $A \leq_{r l p o s}^{P} B$ via machine $M, B \neq \emptyset, B \neq \Sigma^{*}$. Let $B$ be $P$-selective. Then there exists an algorithm that 
runs in time polynomial in the length of the input $x$ and the number of queries in the computation tree of $M$ on $x$, that outputs a set $I$ such that $x \in A \Leftrightarrow I \subseteq B$.

Proof We restate Selman's algorithm for completeness, modified for $\leq_{\text {rlpos }}^{P}$ reductions. Let $f$ be a $P$-selector for $B$. Let $f^{\prime}\left(x_{1}, x_{2}, \ldots, x_{r}\right)=$ $f\left(x_{1}, f\left(x_{2}, \ldots, f\left(x_{r-1}, x_{r}\right), \ldots\right)\right)$. Let $a \in B$ and $b \notin B$.

On input $x$.

1. If $M^{\emptyset}(x)$ accepts let $I=\{a\}$.

2. If $M^{\Sigma^{*}}(x)$ rejects then $I=\{b\}$.

3. Else

Let $Q=T=\emptyset$

Repeat

$I=T$

Simulate $M$ on $x$ with oracle $I$.

$Q=$ set of all queries asked in the above computation.

If $M$ rejects then

begin

$u=f^{\prime}(Q-I)$

$T=I \cup\{u\}$

end

Until $T=I$

Clearly, the above algorithm on input $x$ outputs a set $I$ such that $x \in A \Leftrightarrow I \subseteq B$ (by induction on the number of times the repeat loop is executed). Also since each time the repeat loop is executed the cardinality of $T$ increases at least by 1 , the number of times the repeat loop is executed is at most the number of queries in the computation tree of $M$ on input $x$. Also since each loop runs in time polynomial in the length of $x$ and the number of queries in the computation tree of $M$ on $x$, the whole algorithm runs in time polynomial in $|x|$ and the number of queries in computation tree of $M$ on input $x$.

Corollary 6.8 If $A \leq_{\text {rlpos }}^{P} B, B \neq \emptyset, B \neq \Sigma^{*}$ and $B$ is $P$-selective then for some polynomial $p, A \leq_{m} B$ by a function $g$ computable in time $2^{p(|x|)}$.

Proof Use the above algorithm to get $I$ such that $x \in A \Leftrightarrow I \subseteq B$. Since $\bar{B}$ is $P$-selective, we select an element from $I$ that is most likely to be in $\bar{B}$ (say $y$ ). Now $x \in A \Leftrightarrow y \in B$. 
It is easy to convert a $\leq_{r p o s}^{P}$ computation tree to a $\leq_{r l p o s}^{P}$ computation tree in exponential time. Thus we also have:

Corollary 6.9 If $A \leq_{l p o s}^{P} B, B \neq \emptyset, B \neq \Sigma^{*}$ and $B$ is $P$-selective then for some polynomial $p, A \leq_{m} B$ by a function $g$ computable in time $2^{p(|x|)}$.

Corollary 6.10 If $A \leq_{\text {rpos }}^{P} B, B \neq \emptyset, B \neq \Sigma^{*}$ and $B$ is $P$-selective then for some polynomial $p, A \leq_{m} B$ by a function $g$ computable in time $2^{p(|x|)}$.

For rlptt reductions the number of queries in the computation tree is polynomial in the length of the input; thus we have:

Corollary 6.11 If $A \leq_{r l p t t}^{P} B, B \neq \emptyset, B \neq \Sigma^{*}$ and $B$ is $P$-selective then $A \leq_{m}^{P} B$.

Corollary 6.12 If $A$ is $\leq_{r l p t t}^{P}$ self-reducible and $A$ is $P$-selective then $A$ is in $P$.

Proof From Corollary 6.11 we have $A$ is $\leq_{m}^{P}$ self-reducible. Since any $\leq_{m}^{P}$ self-reducible set is in $P, A \in P$.

Theorem 6.13 [19] For every tally language $A$ there exist sets $B$ and $C$ such that:

1. $B \leq_{p t t}^{P} A \leq_{T}^{P} B$.

2. $C \leq_{t t}^{P} A \leq_{T}^{P} C$.

3. $B \leq_{p t t}^{P} C \leq_{t t}^{P} B$.

4. $B$ is $P$-selective, and

5. $C P$-selective $\Rightarrow C \in P$.

As a corollary we obtain:

Corollary 6.14 Let $A$ be a tally language not in $P$. Then there exist $\leq_{T}^{P}$ equivalent sets $B, C$ such that $C \leq_{t t}^{P} B$ but $C \leq_{r l p t t}^{P} B$. Also $\bar{B} \not_{r l p o s}^{P} B$.

Corollary 6.15 If $E \neq N E$ then there exists sets $B$ and $C$ such that:

1. $B \in N P-P$,

2. $B \leq_{p t t}^{P} C$,

3. $C \leq_{t t}^{P} B$,

4. $C \mathbb{E}_{r l p t t}^{P} B$, and

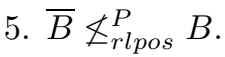

Proof This follows from the above theorem, since if $E \neq N E$ then there are tally sets in $N P-P([4,8]$, see also $[11])$. 
Corollary 6.16 If $E \neq N E$ then there exists a $\leq_{t t}^{P}$ degree in $N P$ that does not consist of a single $\leq_{\text {rlpos }}^{P}$ degree.

Corollary 6.17 If $E \neq N E$ then there exists a $\leq_{r l p t t}^{P}$ degree in $N P-P$ that consists of a single $\leq_{m}^{P}$ degree.

Corollary 6.18 If $E \neq N E \bigcap \operatorname{coNE}$ then there exist sets $B$ and $C$ in $N P$ such that $B \leq_{p t t}^{P} C$ and $C \leq_{t t}^{P} B$ but $C \mathbb{\leq}_{r l p t t}^{P} B$.

\section{Conclusions and Open Problems}

In this paper we defined locally positive reductions as more moderate versions of Selman's (globally) positive reductions. We compared the different locally positive polynomial-time Turing reductions and identified the properties required by positive reductions to obtain the results of Selman - thus enriching the domain in which his results are applicable and delimiting the boundaries of their application.

The two most interesting open problems that remain are (1) whether there exist relativized worlds in which $\leq_{r l p o s}^{P}$ and $\leq_{\text {pos }}^{P}$ are different, and (2) whether

Theorem 6.2 fails for $\leq_{\text {rpos }}^{P}$ or $\leq_{\text {lpos }}^{P}$ in some relativized world. Finally, we note a recent application of our notion of locally robust positive reductions. "Helping" is a notion of fault-tolerant computation acceleration (see $[2,14,16])$. Ko [14] proves that $U P \subseteq P_{1-h e l p}(U P)$, and asks whether equality holds. Recently, Cai, Hemachandra, and Vyskoč [9] have shown that $P_{1-h e l p}(U P)$ is exactly the class of sets locally left positive reducible to $U P$, and, via this characterization, have shown that in relativized worlds, Ko's statement cannot be improved to equality.

\section{Acknowledgements}

We thank William Gasarch and Alan Selman for helpful comments on the paper.

\section{References}

[1] E. Allender. Limitations of the upward separation technique. Mathematical Systems Theory, 24(1):53-67, 1991.

[2] J. Balcázar. Only smart oracles help. Technical Report LSI-88-9, Universitat Politecnica de Barcelona, Barcelona, Spain, 1988.

[3] M. Blum and R. Impagliazzo. Generic oracles and oracle classes. In 28th Annual IEEE Symposium on Foundations of Computer Science, October 1987. 
[4] R. Book. Tally languages and complexity classes. Information and Control, 26:186-193, 1974.

[5] R. Book and K. Ko. On sets truth-table reducible to sparse sets. SIAM Journal on Computing, 17(5):903-919, 1988.

[6] R. Book, T. Long, and A. Selman. Quantitative relativizations of complexity classes. SIAM Journal on Computing, 13(3):461-487, 1984.

[7] R. Book, T. Long, and A. Selman. Qualitative relativizations of complexity classes. Journal of Computer and System Sciences, 30:395-413, 1985.

[8] R. Book, C. Wrathall, A. Selman, and D. Dobkin. Inclusion languages and the Berman-Hartmanis conjecture. Mathematical Systems Theory, 11:1-8, 1978.

[9] J. Cai, L. Hemachandra, and J. Vyskoč. The powers of non-adaptive, faulttolerant, and guarded access to unambiguous computation. In preparation.

[10] J. Hartmanis and L. Hemachandra. Robust machines accept easy sets. Theoretical Computer Science, 74(2):217-226, 1990.

[11] J. Hartmanis, N. Immerman, and V. Sewelson. Sparse sets in NP-P: EXPTIME versus NEXPTIME. Information and Control, 65(2/3):159-181, 1985.

[12] J. Hopcroft and J. Ullman. Introduction to Automata Theory, Languages, and Computation. Addison-Wesley, 1979.

[13] K. Ko. On self-reducibility and weak P-selectivity. Journal of Computer and System Sciences, 26:209-221, 1983.

[14] K. Ko. On helping by robust oracle machines. Theoretical Computer Science, $52: 15-36,1987$.

[15] R. Ladner, N. Lynch, and A. Selman. A comparison of polynomial time reducibilities. Theoretical Computer Science, 1(2):103-124, 1975.

[16] U. Schöning. Robust algorithms: A different approach to oracles. Theoretical Computer Science, 40:57-66, 1985.

[17] A. Selman. P-selective sets, tally languages, and the behavior of polynomial time reducibilities on NP. Mathematical Systems Theory, 13:55-65, 1979.

[18] A. Selman. Analogues of semirecursive sets. Information and Control, 52:36$51,1982$. 
[19] A. Selman. Reductions on NP and P-selective sets. Theoretical Computer Science, 19:287-304, 1982.

[20] G. Tardos. Query complexity, or why is it difficult to separate $\mathrm{NP}^{A} \cup \operatorname{coNP}{ }^{A}$ from $\mathrm{P}^{A}$ by random oracles A. Combinatorica, 9(4):385-392, 1989. 